\title{
Utilization of natural orifice transluminal endoscopic surgery (NOTES) in management of vaginal cuff dehiscence
}

Keywords: dehiscence, natural orifice Transluminal endoscopic surgery, NOTES, vaginal cuff evisceration

\section{Introduction}

Patient is a 29-year-old Gravida1 Para 1001 who presented to our gynecology clinic with complaints of intra-coital bleeding, intermittent spotting and persistent vaginal pain rated 5-6 on a 1-10 pain score of three days duration. Patient was status post Da Vinci single incision laparoscopic surgery (SILS), total laparoscopic hysterectomy (TLH), bilateral salpingectomy, uterosacral ligament suspension (USLS) and cystoscopy for pelvic pain and abnormal uterine bleeding, 10 weeks prior to her presentation. Patient had vaginal intercourse for the first time since her surgery 3 days prior to her presentation and noted bright red vaginal bleeding and pelvic/ vaginal pain after the intercourse.

Clinic exam (as well as examination under anesthesia) showed vaginal cuff dehiscence (Figure 1a \& Figure 1b). Patient was admitted and underwent examination under anesthesia, transvaginal repair of vaginal cuff dehiscence via Natural Orifice Transvaginal Endoscopic Surgery (NOTES) (Figure 2a \& Figure 2b), lysis of adhesions (LOA) (Figure 3a \& Figure 3b), and cystoscopy.
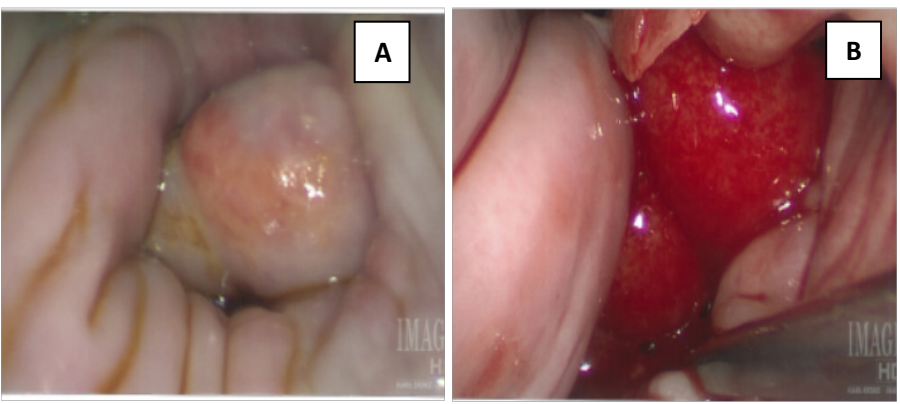

Figure I (A) and (B) Vaginal Cuff defect with bowel epiploic appendages (Omental appendices) of the bowel visible through the vaginal cuff defect.
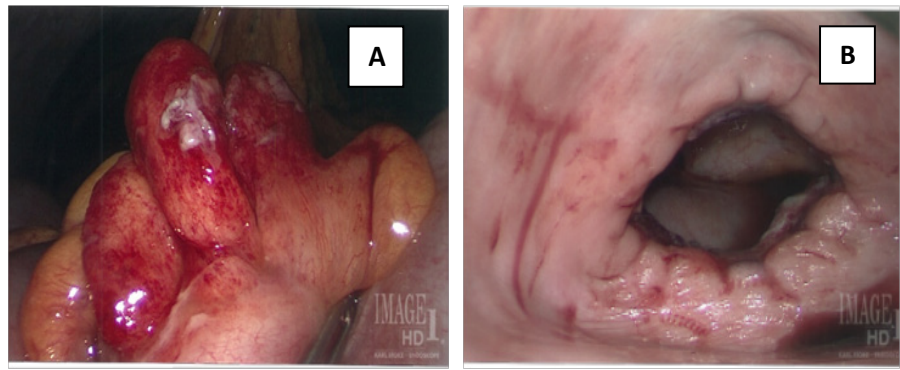

Figure 2 (A): bowel epiploic appendages (Omental appendices) of the bowel were placed back into abdominal cavity, no sign of necrosis noted. (B):Vaginal Cuff Dehiscence.

Patient had an uncomplicated postoperative course and was discharged on postoperative day 1 . She presented for follow-up four weeks postoperatively. Her vaginal cuff was healing appropriately, and she had no complaints.
Volume 10 Issue 3 - 2019

\author{
Shadi Rezai,' Huda Afaneh, ${ }^{2}$ Xiaoming Guan \\ 'Division of Minimally Invasive Gynecologic Surgery, \\ Department of Obstetrics and Gynecology, Baylor College of \\ Medicine, USA \\ ${ }^{2}$ Department of Obstetrics and Gynecology, Hurley Medical \\ Center,Affiliated with Michigan State University College of \\ Human Medicine, USA
}

Correspondence: Xiaoming Guan MD PhD, Division Chief and Fellowship Director, Division of Minimally Invasive Gynecologic Surgery, Department of Obstetrics and Gynecology, Baylor College of Medicine, 665I Main Street, $10^{\text {th }}$ Floor, Houston, Texas, 77030, USA, Tel (832) 826-7464, Fax (832) 825-9349, Email xiaoming@bcm.edu

Received: February 20, 2019 | Published: May 22, 2019
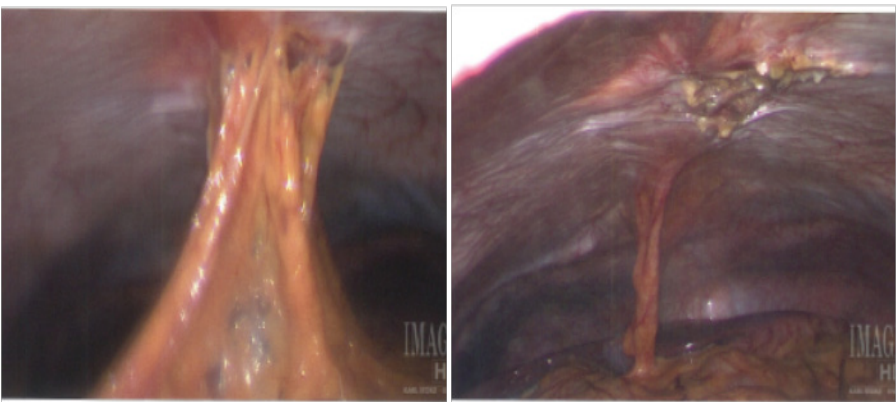

Figure 3 (A):Adhesions bands to umbilicus. (B): s/p Lysis of Adhesions.

\section{Comment}

Vaginal cuff dehiscence and evisceration are not commonly encountered but warrant serious consideration following total hysterectomy. The exact incidence is variable due to the differences among mode of hysterectomy and current data available in the literature which examine patient factors and potential modifiable risks. ${ }^{1,2}$ When closure is the chosen management, there are two approaches for repair, abdominal and vaginal. For patients who have no suspicion for bowel injury or peritonitis, the vaginal approach may be the optimal approach. ${ }^{3}$ In our patient, our initial physical examination showed some redness to the bowel serosa that was concerning for possible bowel compromise (Figure 1a \& Figure 1b). As opposed to evaluating the abdomen laparoscopically, Natural orifice transluminal endoscopic surgery (NOTES) through the vaginal orifice can further optimize the approach to surgery by also examining the abdominal contents directly and safely. ${ }^{4,5}$ This approach may also serve to be more cost-beneficial in the future. ${ }^{6}$ 


\section{Acknowledgments}

Dr. Xiaoming Guan is a speaker for Applied Medical, Rancho Santa Margarita, California. This manuscript was exempt from IRB review at Baylor College of Medicine.

\section{Conflicts of interest}

Other authors did not report any potential conflicts of interests.

\section{References}

1. Nezhat C, Kennedy Burns M, Wood M, et al. Vaginal cuff dehiscence and evisceration: a review. Obstet Gynecol. 2018;132(4):972-985.

2. Hur HC, Donnellan N, Mansuria S, et al. Vaginal cuff dehiscence after different modes of hysterectomy. Obstet Gynecol. 2011;118(4):794-801.
3. Matthews CA, Kenton K. Treatment of vaginal cuff evisceration. Obstet Gynecol. 2014;124(4):705-708.

4. Atallah S, Martin-Perez B, Albert M, et al. Vaginal access minimally invasive surgery (VAMIS): a new approach to hysterectomy. Surg Innov. 2015;22(4):344-347.

5. Liu J, Kohn J, Sun B, et al. Transvaginal natural orifice transluminal endoscopic surgery sacrocolpopexy: tips and tricks. J Minim Invasive Gynecol. 2019;26(1):38-39.

6. Baekelandt J. Total vaginal NOTES hysterectomy: a new approach to hysterectomy. J Minim Invasive Gynecol. 2015;22(6):1088-1094. 